\title{
The genome sequence of the Antarctic bullhead notothen reveals evolutionary adaptations to a cold environment
}

\author{
Seung Chul Shin ${ }^{1}$, Do Hwan Ahn'1,2, Su Jin Kim³ , Chul Woo Pyo ${ }^{4}$, Hyoungseok Lee', Mi-Kyeong Kim¹, \\ Jungeun Lee ${ }^{1}$, Jong Eun Lee ${ }^{5}$, H William Detrich 1 II $^{6}$, John H Postlethwait ${ }^{7}$, David Edwards ${ }^{8,9}$, Sung Gu Lee ${ }^{1,2}$, \\ Jun Hyuck Lee ${ }^{1,2}$ and Hyun Park ${ }^{1,2^{*}}$
}

\begin{abstract}
Background: Antarctic fish have adapted to the freezing waters of the Southern Ocean. Representative adaptations to this harsh environment include a constitutive heat shock response and the evolution of an antifreeze protein in the blood. Despite their adaptations to the cold, genome-wide studies have not yet been performed on these fish due to the lack of a sequenced genome. Notothenia coriiceps, the Antarctic bullhead notothen, is an endemic teleost fish with a circumpolar distribution and makes a good model to understand the genomic adaptations to constant sub-zero temperatures.

Results: We provide the draft genome sequence and annotation for N. coriiceps. Comparative genome-wide analysis with other fish genomes shows that mitochondrial proteins and hemoglobin evolved rapidly. Transcriptome analysis of thermal stress responses find alternative response mechanisms for evolution strategies in a cold environment. Loss of the phosphorylation-dependent sumoylation motif in heat shock factor 1 suggests that the heat shock response evolved into a simple and rapid phosphorylation-independent regulatory mechanism. Rapidly evolved hemoglobin and the induction of a heat shock response in the blood may support the efficient supply of oxygen to cold-adapted mitochondria.

Conclusions: Our data and analysis suggest that evolutionary strategies in efficient aerobic cellular respiration are controlled by hemoglobin and mitochondrial proteins, which may be important for the adaptation of Antarctic fish to their environment. The use of genome data from the Antarctic endemic fish provides an invaluable resource providing evidence of evolutionary adaptation and can be applied to other studies of Antarctic fish.
\end{abstract}

\section{Background}

Antarctic fish have experienced extraordinary evolutionary episodes since the cooling of the Southern Ocean to the freezing point of seawater $\left(-1.9^{\circ} \mathrm{C}\right)$ about 34 million years ago after the opening of the Drake passage and the establishment of the Antarctic Circumpolar current, which led to thermal isolation and widespread glaciation of Antarctica [1,2]. Particularly in this environment, adaptations occurred including an antifreeze glycoprotein

\footnotetext{
*Correspondence: hpark@kopri.re.kr

'Division of Polar Life Sciences, Korea Polar Research Institute, Yeonsu-gu, Incheon 406-840, South Korea

${ }^{2}$ Polar Sciences, University of Science \& Technology, Yuseong-gu, Daejeon 305-333, South Korea

Full list of author information is available at the end of the article
}

gene that evolved from a duplicated trypsinogen gene [3-5], cold-efficient microtubule assembly [6,7], loss of an inducible heat shock response [8-10], and changes in membrane fluidity [11]. The Channichthyidae (white-blooded icefish) clade of Notothenioids even lost functional hemoglobin, myoglobin, and the ability to make red blood cells $[2,12,13]$. The history of these evolutionary episodes can likely be decoded from explorations of the genomes of Antarctic fish and their compensatory adaptations to their near-freezing environment.

Waters of the Antarctic continental shelf and upper slope contain 222 species of fish from 19 families. The Notothenioids, a perciform group, account for $45.5 \%$ of the species [14]. In the high latitude (71S-78S) embayments of the Ross and Weddell Seas, Notothenioids dominate 
Antarctic fish fauna and represent $77 \%$ of the species diversity. It would be $92 \%$ of the number of individuals, and $91 \%$ of the biomass [15]. Ninety-seven percent of Antarctic Notothenioid fish are endemic [16]. Notothenia coriiceps (Richardson, 1844) is one of the major Antarctic fish for studies of adaptation in the Southern Ocean [3,17-19]. $N$. coriiceps is highly abundant in near-shore Antarctic waters and may have a circumantarctic distribution [20]. Here we discuss the sequencing and analysis of the genome of the Antarctic bullhead notothen, $N$. coriiceps, and report transcriptome analysis from RNA-seq experiments conducted to explore temperature challenges involved in cold-adapted evolution. We sequenced the genome of an Antarctic bullhead notothen, $N$. coriiceps, applying a whole genome shotgun approach to a total of $84.5 \times$ coverage for its estimated genome size of $637 \mathrm{Mb}$ to understand these evolutionary mechanisms. This report illuminates evolutionary trajectory of some major life-history traits of these Antarctic fish, provides important clues for ecological and population studies designed to address issues of Antarctic biota, and contributes a reference genome for use in future comparative studies of Antarctic adaptations.

\section{Results}

\section{Sequence and assembly}

We sequenced genomic DNA extracted from a single Notothenia coriiceps collected at northern Antarctic Peninsula. We used three sequencing platforms: Illumina HiSeq2000, GS-FLX, and Pacbio RS with coverage of $78.6 \times, 2.0 \times$, and $3.9 \times$, respectively. Initial hybrid assemblies were performed using the Celera Assembler with Illumina short reads and 454 reads [21] (Additional file 1: Tables S1 and S2). A total of 25,794 assembly gaps were filled with Illumina reads and error-corrected continuous long reads (CLR) generated from Pacbio $R S$ [22,23] (Additional file 1: Table S3 and Additional file 2: Figure S1). A total of 18,400 gaps were filled with Illumina reads using Gapfiller (Ver. 1.9) and 7,394 gaps were closed with CLR reads using PBjelly (Ver. 12.9.14). The final assembly consisted of 38,062 scaffolds that comprised 100,606 contigs spanning $637 \mathrm{Mb}$ with remaining unclosed gaps of roughly $13.1 \mathrm{Mb}$ (2.1\% of the total scaffold sequence). To validate the final assembly accuracy of the scaffolds, we sequenced and assembled bacterial artificial chromosomes (BACs) clones using GS-FLX. Six sequenced BAC clones were aligned to the scaffolds, and $99 \%$ of the total BAC clones were identical to the assembled scaffolds (Additional file 2: Figure S2). The final assembly had an N50 contig size of $11.6 \mathrm{~Kb}$ and an N50 scaffold size of 219 $\mathrm{Kb}$, and the largest scaffold was $28 \mathrm{Mb}$ (Table 1 ).

\section{Genome annotation}

For gene prediction, we used $36 \mathrm{~Gb}$ of RNA sequencing data from seven tissues (brain, skin, egg, kidney, muscle, stomach, and blood) and $300 \mathrm{Mb}$ error-corrected CLR from three tissues (egg, skin, and muscle) (Additional file 1: Tables S4 and S5), and the MAKER annotation pipeline approach using both evidence-based and ab initio methods [24] resulting in a final gene set of 32,260 protein-coding genes. A total of 29,045 of these protein-coding genes were assigned preliminary functions with BLASTp, and we could assign Gene Ontology (GO) terms to 19,556 (60.57\%) predicted genes based on BLASTp results and InterproScan, encompassing biological processes (14,602 (45.22\%)), cellular components $(12,511(38.75 \%))$, and molecular functions (15,972 (49.47\%)) (Additional file 2: Figure S3). Enzyme commission (EC) was obtained for 3,465 proteins (Additional file 1: Table S6 and S7). Annotated genes contained an average of 6.65 exons, with an average mRNA length of 1,478 bp and CDS length of 1,063 bp. The de novo repeat prediction showed that repeat sequences accounted for $18.15 \%$ of the assembled $N$. coriiceps genome (Additional file 1: Table S8) and 529 tRNA were also predicted (Additional file 1: Table S9).

\section{The evolution of gene families in $N$. coriiceps}

Gene families are groups of homologous genes that possess highly identical structures and similar functions. These families vary in gain or loss of genes, making the size different among gene families through evolution [25-27]. To identify the evolution of $N$. coriiceps gene families, we investigated the size differences between 18,131 gene families with at least two genes across six fish of interest (Danio rerio, Gasterosteus aculeatus, Takifugu rubripes, Tetraodon nigroviridis, Gadus morhua, and $N$. coriiceps) (Figure $1 \mathrm{~A}$ and B). We were able to identify the largest number of gene family contractions (5,495 gene families) and the average reduction (0.344), which means number of gene lost per family, in the $N$. coriiceps lineage. The lineage $D$. rerio has the largest number of gene family expansions $(4,715)$ among these six fish. In the likelihood approach to studying gene family evolution, gene families evolving at significant rates of gain and loss than the genome wide average could exhibit higher expansions or contractions [27]. From the 18,131 gene families, 82 showed significant expansions or contractions among six fishes at $P<0.0001$ [26-29]. At this significance level, only one family is expected by chance (a false discovery rate $=0.02 \%$ ); finally we identified 32 families showing significantly difference in $N$. coriiceps lineage. However, we were not able to identify significant expansions in $N$. coriiceps, but only significant contractions occurred in 32 gene families (Additional file 3).

To investigate selective pressures on protein-coding genes of $N$. coriiceps, we determined the $\mathrm{dN} / \mathrm{dS}$ ratio (the ratio of the rate of non-synonymous substitutions to the rate of synonymous substitutions) of 8,974 orthologs 
Table 1 Global statistics of the $N$. coriiceps genome assembly

\begin{tabular}{|c|c|c|c|c|}
\hline Sequencing platform & \multicolumn{2}{|c|}{ Insert size } & Total data $(\mathrm{Mb})$ & Sequence coverage $(x)$ \\
\hline Illumina paired-end & \multicolumn{2}{|c|}{$150,300,350,500,600 \mathrm{bp}$} & 47,163 & 78.6 \\
\hline GS-FLX Mate-pair & \multicolumn{2}{|c|}{ Single, 3, 8, $20 \mathrm{~kb}$} & 1,185 & 2.0 \\
\hline PacBioRS & \multicolumn{2}{|c|}{ Continuous Long Read } & 2,318 & 3.9 \\
\hline Assembly results & Number & $\mathrm{N} 50(\mathrm{~kb})^{\mathrm{a}}$ & $M L(k b)^{b}$ & Size (Mb) \\
\hline Contig & 100,606 & 11.6 & 226.8 & 622 \\
\hline Scaffold & 38,062 & 219.1 & $28,796.7$ & 637 \\
\hline Annotation & \multicolumn{2}{|l|}{ Number } & Total length (kb) & Percentage of genome \\
\hline Genes & \multicolumn{2}{|l|}{32,260} & 47,712 & 7.5 \\
\hline Repeats & & & 115,561 & 18.15 \\
\hline
\end{tabular}

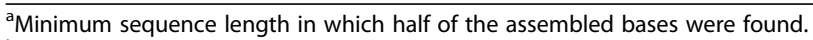

${ }^{\mathrm{b}}$ Maximum length.

in six fish (Figure 1A, Additional file 1: Table S10 and Additional file 2: Figure S4). Orthologs showing poor alignment were removed, and those with high synonymous substitution rates (over 3) and excessive transition/transversion ratios (over 10) were also removed. Finally, the dN/dS of 5,039 orthologs were determined. The average $\mathrm{dN} / \mathrm{dS}$ ratio of $N$. coriiceps (0.133) was significantly higher than that of the other five fish (range: 0.050 to 0.115) (Figure 1C). We interpret this comparison to indicate a high level of selective pressure caused by the harsh Antarctic environment. To determine which functional gene categories evolved most rapidly, we selected 505 rapidly evolving genes with $\mathrm{dN}$, as an indicator to categorize fast and slow evolving orthologs, in the top 10\% of 5,039 genes in $N$. coriiceps, then analyzed them for statistically over-represented genes in the Gene Ontology [30-32] (Additional file 1: Table S11 and Additional file 4). Seventeen GO terms (included 46 genes) were significant in GO enrichment analysis using selected 505 rapidly evolving genes (Figure 1D and Additional file 1: Table S12). The average $\mathrm{dN} / \mathrm{dS}$ ratio (0.294) of 46 genes in $N$. coriiceps was statistically higher than the orthologs of these 46 genes in the other five fish (approximately 0.061 to 0.150). It is noteworthy that $13 \mathrm{GO}$ terms among enriched $17 \mathrm{GO}$ terms were associated with mitochondria (Additional file 1: Table S12). We focused on 20 mitochondrial protein-coding genes that were enriched in 13 GO terms (Additional file 1: Table S12). Results showed that the average $\mathrm{dN} / \mathrm{dS}$ ratio $(0.320)$ for this set of $20 N$. coriiceps mitochondrial genes was also statistically higher than other fish mitochondrial genes (approximately 0.0630 .147 ) (Figure 1E). Our observation that most GO terms linked to rapidly evolved mitochondrial genes in $N$. coriiceps might be functions correlated with high thermal sensitivity in Antarctic notothenioids [33-35]. Other GO terms significantly enriched among rapidly evolving genes were glutathione transferase activity (GO:0004364), rhodopsin kinase activity (GO:0050254), and oxygen transporter activity (GO:0005344) in molecular function, and MHC class I protein complex (GO:0042612) in cellular component. Oxygen transporter activity (GO:0005344) among significant enriched GO terms including alpha and beta globins might be also associated with mitochondria through supplying oxygen for their oxidative phosphorylation.

We also investigated whether the rapidly evolving genes as defined by $\mathrm{dN}$ are specific to the $N$. coriiceps lineage and whether the results have been due to positive selection or relaxation of selection pressure. We used a branch-specific model, and identified that the $\mathrm{dN} / \mathrm{dS}$ of 117 genes were significantly different from the rest of the phylogenetic tree of six fish. Seventy-two genes (including 10 mitochondrial genes) among 117 genes (including 14 mitochondrial genes) were under significant positive selection (Additional file 1: Table S11), and also the oxidative phosphorylation (GO:0006119) of GO term was statistically represented in the GO enrichment test for genes under positive selection.

\section{Heat shock factor in N. coriiceps}

Although the heat shock response (HSR), a defense mechanism against thermal stress, is inducible in most animals, Antarctic notothenioid fish have been reported to lack an inducible HSR [12,31]. In contrast, HSR proteins in Antarctic fish are constitutively expressed, presumably to mitigate cold denaturation of proteins [10,36-39]. To identify whether the loss of gene affects the constitutive HSR expression with the cold denaturation of proteins [40], we investigated the genes related to the regulation of the HSR in the $N$. coriiceps, and identify that HSR-related genes were well-conserved in their draft genome and their gene expressions were also identified by reverse transcriptase PCR (rt-PCR) in normal condition or in stress condition (Figure 2A). In vertebrate HSR, the heat shock factor 1 (HSF1) gene is known as the master regulator. Therefore, we investigate whether the functional domain of HSF1 was well conserved. As a result, HSF1 lost its phosphorylation-dependent sumoylation motif (PDSM), which is essential for repressing its transactivation capacity 


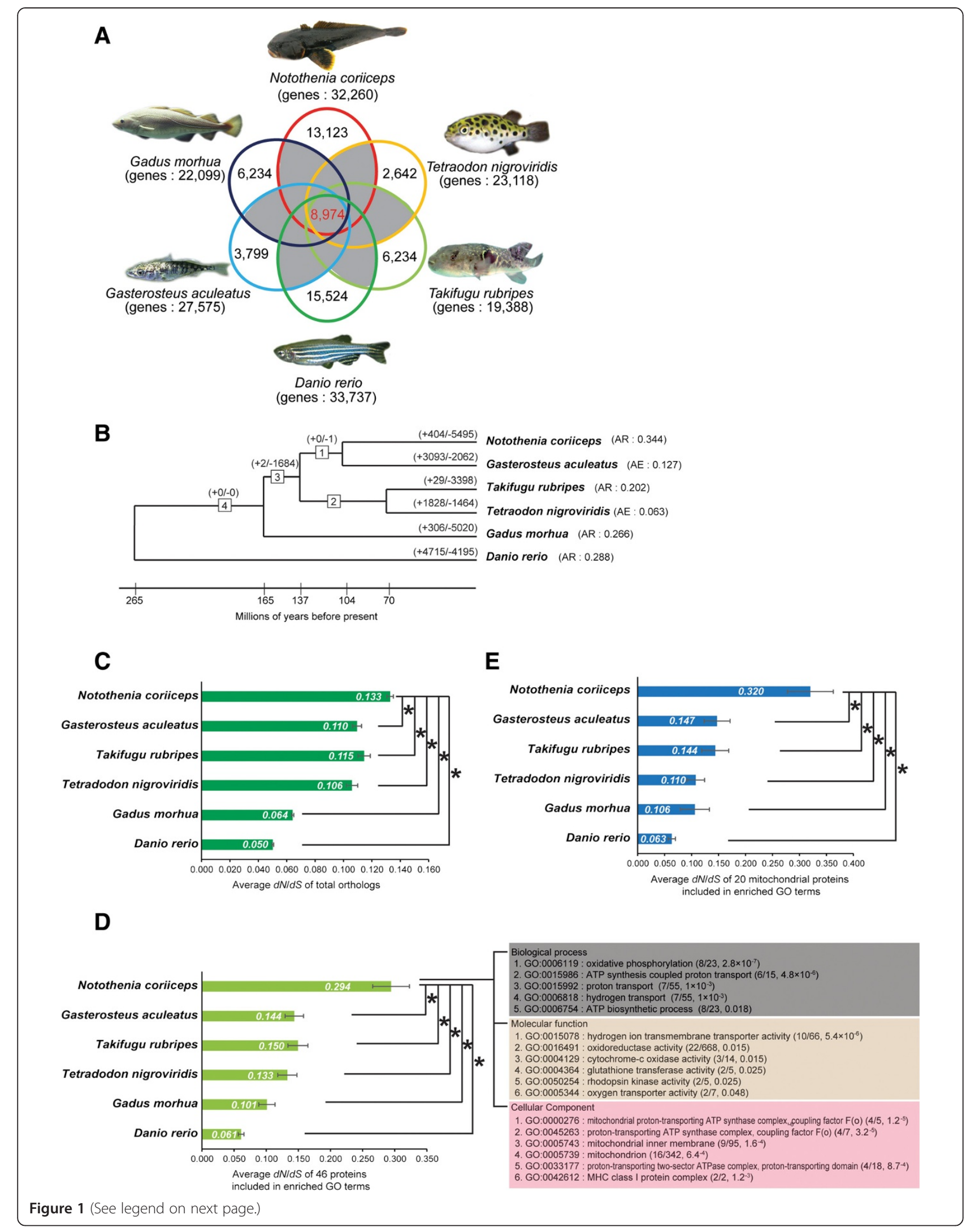


(See figure on previous page.)

Figure 1 Genome-wide analysis of protein-coding genes in $\mathbf{N}$. coriiceps. (A) Venn diagram displaying the overlap in gene families in six fish species. A total of 18,131 gene families that are included in gray background were used to analyze gain and loss of gene in six fish. (B) Lineage-specific genes expansion and contraction among six fish. The numbers in boxes are identifiers for internal branches of the phylogeny. Numbers on each branch denote the number of gene gains (+)/losses (-). AE and AR denote average expansion family (mean number of genes gained) and average reduction family (mean number of genes lost), respectively. (C) The average $\mathrm{dN} / \mathrm{dS}$ of 5,039 orthologs were determined. Bar charts show the average $\mathrm{dN} / \mathrm{dS}$ values for six fish species. Data were analyzed using an analysis of variance (ANOVA) followed by Bonferroni post hoc test; values represent mean \pm SEM $(* P<0.001)$. (D) In a GO enrichment test among the rapid-evolving genes with $\mathrm{dN}$ in the top $10 \%, 17 \mathrm{GO}$ terms including 46 genes were significantly enriched in N. coriiceps. The average dN/dS of 46 proteins included in enriched GO terms. Bar charts show the average dN/dS values for six fish species. Data were analyzed using an analysis of variance (ANOVA) followed by Bonferroni post hoc tests; values represent mean \pm SEM

( $\left.{ }^{*} P<0.001\right)$. (E) The average $\mathrm{dN} / \mathrm{dS}$ ratio of 20 mitochondrial proteins included in enriched $\mathrm{GO}$ terms. Bar charts show the average $\mathrm{dN} / \mathrm{dS}$ values for six fish species. Data were analyzed using an analysis of variance (ANOVA) followed by Bonferroni post hoc test; values represent mean \pm SEM ( $* P<0.001$ ).

(Figure 2B and Additional file 2: Figure S5). Phosphorylation of the serine residue in the PDSM (KxExxSP) is a prerequisite for conjugation of a small ubiquitin-related modifier peptide (SUMO) to a single lysine residue in HSF1. When maximal HSF1 activity is required, desumoylating enzymes remove this modification from $H S F 1$ [41-44] (Figure 2B and C). We found that the serine residue of PDSM in HSF1 was substituted with asparagine in $N$. coriiceps and in other Antarctic fish as well including the icefish Chaenocephalus aceratus and the dragonfish Parachaenichthys charcoti) (Additional file 2: Figure S5). In response to thermal stress, the DNA-binding and transactivation capacity of $H S F 1$ are coordinately regulated through multiple post-translational modifications (PTMs), protein-protein interactions and subcellular localization [41]. Loss of this sumoylation site of PDSM (Ser303 to Asn303) would allow for maximal activation in response to heat shock stress with simple methods in the genomic context of $N$. coriiceps (Figure 2C) [41,43].

\section{Heat shock response in $N$. coriiceps}

To address the question of response to heat shock, raised by our finding of the loss of the sumoylation site in the $N$. coriiceps HSP1 protein, we investigated the HSR in these fish. $N$. coriiceps is a stenothermal fish that can survive only within the range of $-2.5^{\circ} \mathrm{C}$ to $6.0^{\circ} \mathrm{C}$ [45]. When we exposed $N$. coriiceps to heat shock $\left(4^{\circ} \mathrm{C}\right)$ for 48 h, 32 genes (including HSP70, HSP4O, and Heat shock protein ssb1) were significantly upregulated more than two-fold based on the RNA-seq analyses (Figure 3A, Additional file 1: Tables S13 and S14). We confirmed that HSR-related genes were upregulated in the whole blood sample using qPCR (Figure 3B). The expression level of HSP70 were positively correlated with increasing exposure to high temperatures up to $48 \mathrm{~h}$ (Figure 3C) and declined to baseline after $24 \mathrm{~h}$ of recovery from heat shock stress. So far, heat shock proteins are known to be constitutively expressed in gill and liver tissues from Antarctic notothenioid fish [8,10], and we could identify that the HSP7O was also constitutively expression, but not induced in liver or other tissues (brain, skin, kidney, and spleen) (Figures 2A and 3B). This was the first observation that the expression of HSR-related genes was induced under heat stress in the whole blood sample. Because cold stress, as well as heat stress, also can denature protein structures $[37,39,46]$, we investigate whether the expression of several HSP genes is induced under cold shock stress at $-2^{\circ} \mathrm{C}$. We found that 46 genes were significantly upregulated more than two-fold under cold stress and 13 genes were also upregulated under heat shock stress, including HSP genes (Figure 3A,B,C, and Additional file 1: Table S15). Based on a GO enrichment test among genes upregulated more than two-fold under heat or cold stresses, the majority of enriched GO terms were shared between the two stressors (Additional file 1: Tables S16, S17 and Additional file 2: Figure S6). Shared GO terms resulting from shared genes upregulated under opposite stresses, such as heat and cold, were relevant in terms of protein stability. The induced HSR in Antarctic fish under thermal stress indicates that proteins were stable under normal conditions in blood. Based on tissue-specific gene expression analyses, genes related to the unfolded protein response (UPR) [47-49] with $H S P 70, F K B P$, the $78-\mathrm{kDa}$ glucose-regulated protein precursor (GRP78), IRE1, and transcription factor X-box binding protein $1(X B P 1)$, were also downregulated in whole blood compared to other tissues (brain, skin, liver, kidney, intestine, and spleen) (Additional file 2: Figure S7). The relative expression levels of genes related to the UPR support the stable states of blood proteins.

\section{Discussion}

The evolution of gene families in $N$. coriiceps

We investigated the size differences in gene family and calculated the $\mathrm{dN} / \mathrm{dS}$ value to uncover evidence for evolution in Antarctic fish. We found significant contractions in 32 gene families, but could not identify significant expansions based on gene family evolution analysis. Although several genes (three solute carrier families and two glutamate receptors) of similar functions were found in contracted gene families (Additional file 3), 


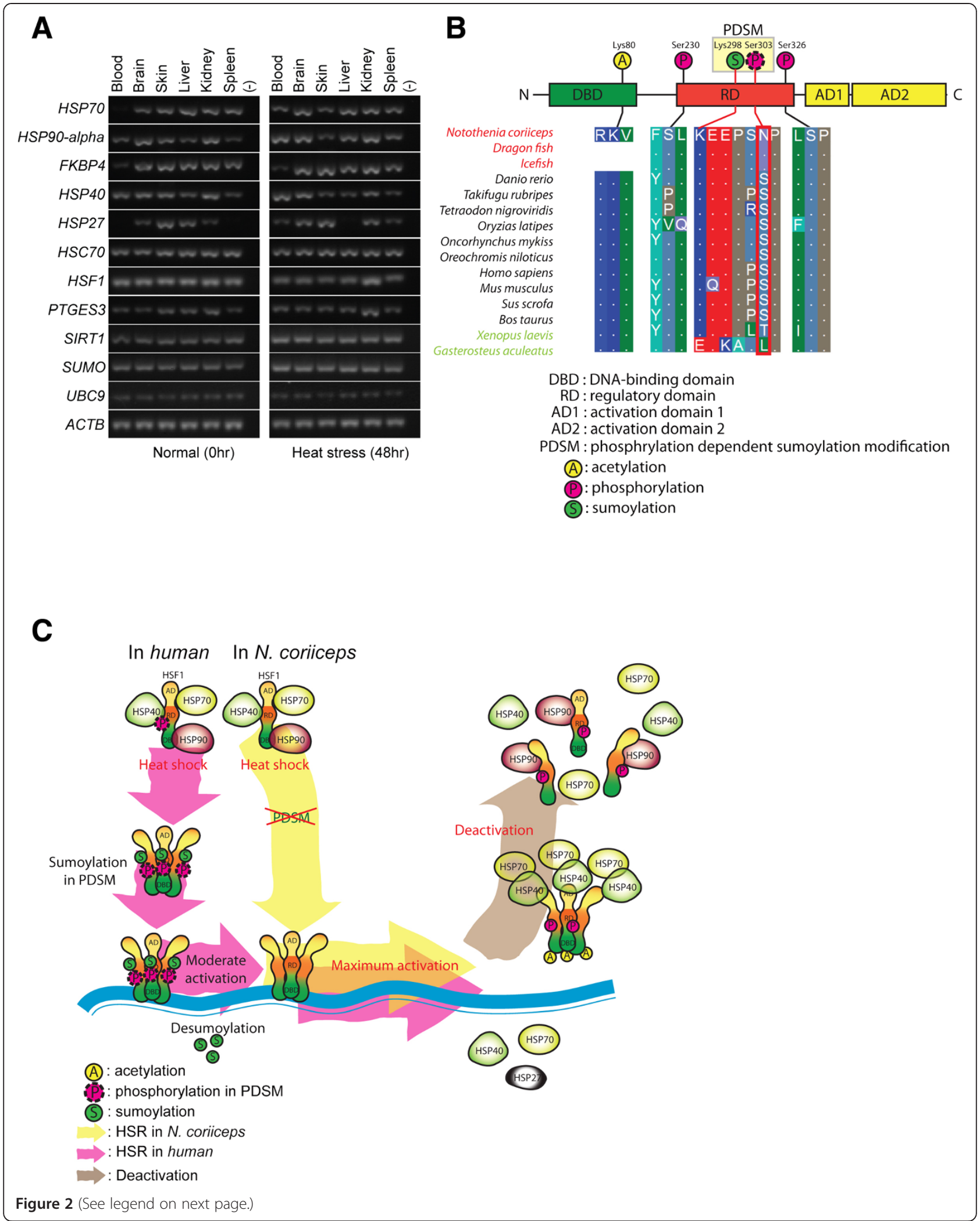


(See figure on previous page.)

Figure 2 Heat shock response in $\mathbf{N}$. coriiceps. (A) Tissue specific expression of HSR-related genes in various tissues. Multi-chaperone complexes of HSP90 (FKBP and PTGES), SIRT1 (NAD-dependent decetylase sirtuin-1; SIRT1 is negatively related to the acetylation of HSF1 in humans), SUMO, HSP90-alpha, and UBC9 were expressed in blood and other tissues. The SUMO-cconjugation enzyme ubiquitin carrier 9 (UBC9) discriminates between the phosphorylated and non-phosphorylated PDSM of HSF1. (B) HSF1 and its post-translational modification sites in N. coriiceps. Serine corresponding to ser303 of human HSF1 was substituted with asparagine. This site is responsible for sumoylation of lys298 of PDSM in human hsf1. In Xenopus sp. and G. aculeatus, other amino acids were identified at the site corresponding to ser303 of human HSF1. All sequenced mammals and most fish have serine at this site. This substitution of serine to asparagine was also identified in icefish and Dragon fish in the Antarctic Ocean. (C) Components responsible for the HSR in N. coriiceps and its regulation.

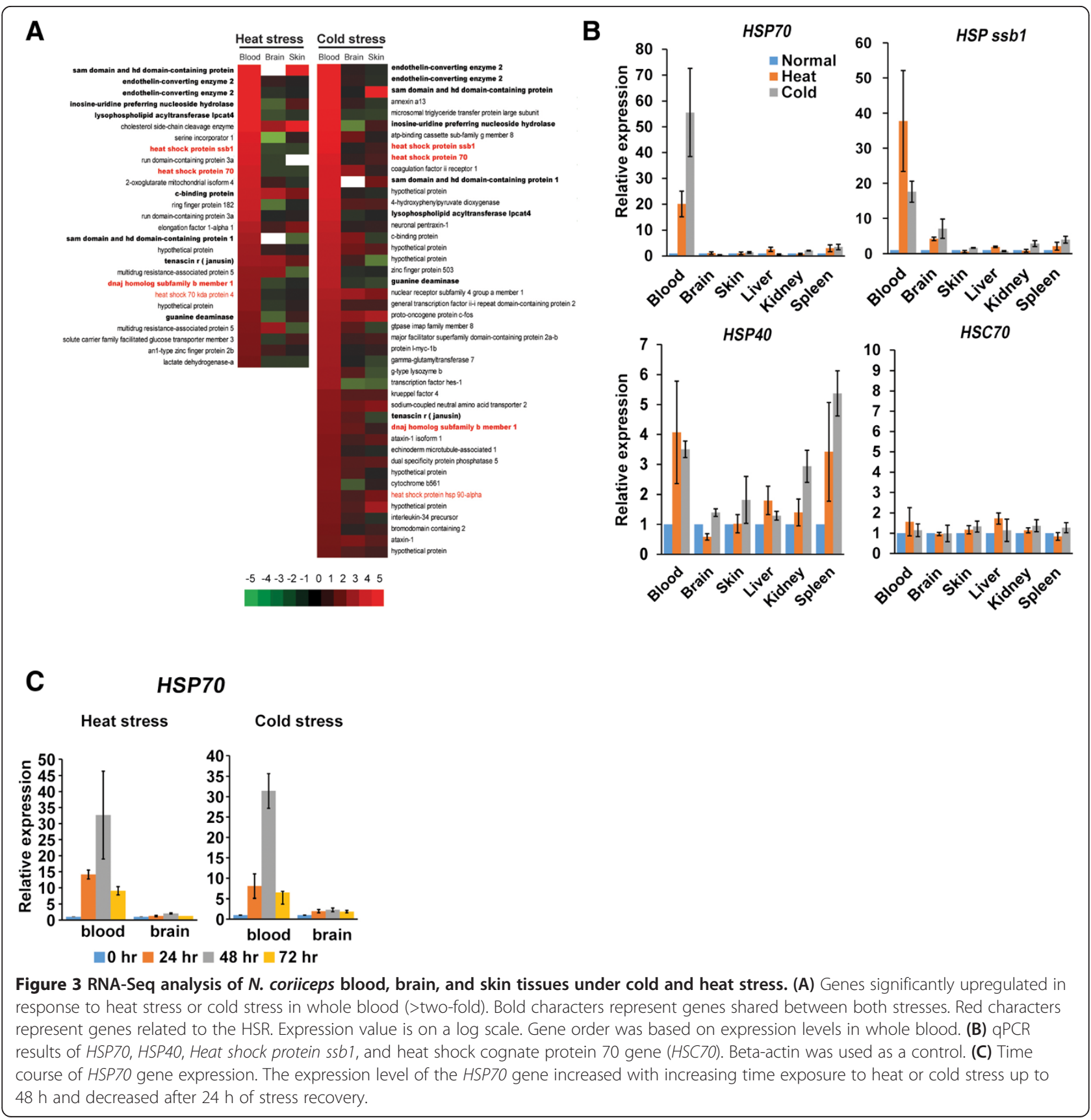


we could not find significant GO terms in GO enrichment tests for the group of contracted gene families. We confirmed that $N$. coriiceps has the largest contractions of gene families among six fish. In $\mathrm{dN} / \mathrm{dS}$ analyses comparing orthologs, our observation that the average $\mathrm{dN} / \mathrm{dS}$ ratio of $N$. coriiceps genes was significantly higher than that of five other fish are supporting the conclusion of strong selection pressure affected the average $\mathrm{dN} / \mathrm{dS}$ ratio of $N$. coriiceps (0.133). The constant low temperature around $1^{\circ} \mathrm{C}$ and higher oxygen solubility in the Southern Ocean would likely be strong selective factors on Antarctic fish in the 34 million years since Antarctica started to cool.

In $\mathrm{GO}$ enrichment tests with the most rapidly evolving $10 \%$ of $N$. coriiceps genes, we confirmed that most enriched GO terms were related with mitochondria. The average $\mathrm{dN} / \mathrm{dS}$ ratio of 20 genes encoding mitochondrial proteins in $N$. coriiceps was significantly higher than that found in other five fish. Ten genes were directly related to oxidative phosphorylation; six genes encoded subunits of ATP synthase, one gene encoded a subunit of mitochondria complex III, and three genes encoded subunits of mitochondria complex IV. This observation indicates that rapid evolution in mitochondrial proteins might be related to adaptation under cold environment. Investigations on mitochondrial function of Antarctic fish showed that oxygen consumption rates and the coupling efficiency between electron transport and ATP synthesis were more sensitive to temperature than temperate fish [33-35]. The Arrhenius break temperature (ABT) reflects the adaptation temperature of the species, and it is the temperature at which there is a discontinuity in the slope of an Arrhenius plots of $\mathrm{O}_{2}$ consumption versus temperature. ABT for mitochondria of Antarctic fish is around $12^{\circ} \mathrm{C}$ much less than $20^{\circ} \mathrm{C}$ from several taxa of marine invertebrates and fish [33-35]. The temperature of acceptor control ratio (ACR), which is the ratio max rate adenosine diphosphate (ADP) induced $\mathrm{O}_{2}$ consumption to the basal rate in the absence of ADP, began to decrease also reflects the adaptation temperature. ACR decreased at approximate $18^{\circ} \mathrm{C}$ in the mitochondria of Antarctic fish in contrast to about $35^{\circ} \mathrm{C}$ in temperate fish [35]. Low $\mathrm{ABT}$ and the decrease of $\mathrm{ACR}$ at low temperature in the mitochondria of Antarctic fish is likely an adaptation to the cold and thermostable Southern Oceans, and might affect the rapid death of stenotherm, Antarctic fish, at a temperature around $10^{\circ} \mathrm{C}$ [34]. Oxygen transporter activity, including alpha and beta globins, among enriched GO terms were also related to the function of oxidative phosphorylation by efficiently supplying oxygen to mitochondria. Their rapid evolution might influence the phenomenon of thermal sensitivity of mitochondrial function in Antarctic fish and might be helpful to interpret the thermal limit of metabolic acclimation [50,51].

\section{Loss of PDSM in HSF1 and heat shock response in blood sample of $N$. coriiceps}

In most organisms, heat shock responses are mediated mainly by heat shock factors. In the presence of stress, HSF1 complex dissociates to HSP90, HSP40, and HSP70, after that HSF1 have trimerized [52]. Trimeric HSF1 localizes to the nucleus and activates the transcription of target genes. During this process, HSF1 undergoes extensive PTMs in regulatory domain [41]. In humans, HSF1 contains a regulatory domain with three phosphorylation sites and one phosphorylation-dependent sumoylation motif (PDSM) [42], and sumoylation of HSF1 is inversely related to HSF1 activity [43,44]. HSF1 is maximally activated in the absence of sumoylation [43] (Figure 2C). During the attenuation phase, the transactivation of HSF1 is negatively regulated by elevated levels of both HSP40 (DNAJB1) and HSP70 [52]. The DNA-binding activity of HSF1 is regulated by acetylation of HSF1, and the expression of $\mathrm{NAD}^{+}$-dependent sirtuin (SIRT1) is negatively associated with acetylation of HSF1 (Figure 2B and C) [52]. We found that the major proteins regulating the HSR were expressed in blood and other tissues based on both RNA-seq and rt-PCR (Figure 2A) and the DNA-binding activity of HSF1 is reported in hepatocytes from Trematomus bernacchii (a common Antarctic notothenioid species) [36]. Sequence alignments also showed that HSF1 of $N$. coriiceps contains conserved DNA binding domains, regulatory domains, and site of sequences related to trimerization. However, the HSF1 of N. coriiceps does not contain an intact PDSM in the regulatory domain, but Asn is substituted for Ser in PDSM (KxExxSP) (Additional file 2: Figure S5). Consequently, HSF1 of $N$. coriiceps does not sumolyated by PTMs, which means the transactivation of HSP1 was not repressed by sumoylation. The absence of sumoylation in HSF1 maximizes more easily activated of HSF1 in the presence of stress (Figure 2C) [43]. We hypothesize that the HSR in Antarctic notothenioids is not constitutively activated to repair protein denaturation in constant cold environment, but readily responsive to the environment. Based on our transcriptomic experiments examining heat shock protein expression in various tissues subjected to thermal stress (Figure 3A), induction of the HSR was consistent with our hypothesis. The HSR in N. coriiceps has retained the ability to increase the expression of heat shock proteins (HSP70, HSP ssb1, and HSP40) at the transcriptional level in whole blood of $N$. coriiceps in response to acute thermal stress. Molecular evolution of alpha and beta globin, which comprise $21.2 \%$ of total transcripts in whole blood samples (Additional file 1: Table S18), might be related to this hypothesis. Because hemoglobin makes up about $40 \%$ of red blood cells in several red-blooded notothenioids [53], their rapid evolution might affect protein stability. Together with the presence of antifreeze glycoprotein in blood, which originated primarily from the 
exocrine pancreas and the stomach [3-5], our observation that HSR occurred in blood of $N$. coriiceps might be one of the evolution strategies to supply adequate oxygen in cold environment.

\section{Tissue-specific gene expression patterns related to HSR and UPR}

Tissue-specific gene expression patterns related to the HSR showed that the expression of FKBP and HSP70 were decreased in whole blood samples compared to other tissues in normal condition (Additional file 2: Figure S10). The heat shock response is known to occur in the cytosol, so we also examined genes associated with UTR in the endoplasmic reticulum (ER) (Additional file 2: Figure S7) [47,49]. GRP78, IRE1, and XBP1 were downregulated in blood. The UPR is typically triggered in response to accumulation of misfolded proteins in the lumen of the ER, after GRP78 is released from IRE1 to support proper protein folding [49]. IRE1 through autophosphorylation activates its ribonuclease domain and catalyzes the excision of unconventional introns from ubiquitously expressed XBP1 [48]. This excision causes a frame shift in the XBP1 coding sequence, resulting in the production of the 376-amino acid XBP1 protein. Active XBP1 upregulates genes involved in UPR $[47,49]$. These results support our conclusion that blood proteins in cytosol and in ER have the stability which do not induced the constitutive HSR.

\section{Conclusions}

In this study, we provide the first annotated genome of an Antarctic species that dominates the fish fauna of the Southern Ocean and shows remarkable adaptations to cold temperatures. The genome sequence of $N$. coriiceps increases our understanding of the evolution trajectory of some major life-history traits of these Antarctic fish. We demonstrated that $N$. coriiceps have rapidly evolved mitochondrial proteins and hemoglobin, and have preserved the HSR in blood. Our observations were associated with oxidative phosphorylation in aerobic cellular respiration and might make a contribution to adapt to an extremely cold environment through the proper function of aerobic cellular respiration. Our study provides a reference genome for use in future comparative studies of Antarctic adaptations and can be applied to ecological and population studies of Antarctic biota.

\section{Materials and methods}

Ethics statement

This study including sample collection and experimental research conducted on these animals was according to the law on activities and environmental protection to Antarctic approved by the Minister of Foreign Affairs and Trade of the Republic of Korea.

\section{DNA library construction and sequencing}

$N$. coriiceps (length $35 \mathrm{~cm}$ ) were collected from depths of 20 to $30 \mathrm{~m}$ in Marian Cove, near King Sejong Station, on the northern Antarctic Peninsula (62 $\left.14^{\prime} \mathrm{S}, 58^{\circ} 47^{\prime} \mathrm{W}\right)$ in January 2012 using the hook-and-line method, and water temperatures were monitored at $1.6 \pm 0.8^{\circ} \mathrm{C}$ in January 2012. High-molecular-weight genomic DNA from $N$. coriiceps was extracted using the Gentra Puregene Blood Kit (Qiagen). For Illumina Hiseq 2000 sequencing, five library types were constructed with 150, 300, 350, 500 , and $600 \mathrm{bp}$ sheared genomic DNA, and subsequently prepared using the standard Illumina sample preparation methods. Mate-pair libraries (3, 7, and $20 \mathrm{~kb}$ ) for the GS-FLX titanium apparatus were prepared for scaffolding, and sequencing was performed according to the manufacturer's instructions (Additional file 1: Table S1). All sequencing processes were performed by DNA Link, Inc. (Additional file 1: Table S2).

\section{Genome assembly using Celera assembly}

Hybrid assemblies were performed using the Celera Assembler (Ver. 7.0) with Illumina short reads and 454 reads [21]. Prior to assembly, Illumina reads were trimmed using the FASTX-Toolkit (Ver. 0.0.11) [54] with the parameters -t 20, -1 70, and -Q 33, after which a paired sequence from trimmed Illumina reads was selected. Finally, read data with 110 -fold coverage were obtained. Among the final read data, $74 \times$ trimmed Illumina reads with various insert sizes (150, 350, 500, and $600 \mathrm{bp}$ ) were randomly selected due to memory limitation on the available linux machine, and converted to the FRG file format (required by the Celera assembler) using FastqToCA. Using sffToCA, $1.8 \times 454$ reads were converted to the FRG file format by removing a linker sequence from 454 reads generated using GS-FLX. Assembly was performed on a 96-processor workstation with Intel Xeon X7460 $2.66 \mathrm{GHz}$ processors and 1 terabyte RAM with the parameters overlapper $=$ ovl, unitigger $=$ bogart, utgGraphErrorRate $=0.03$, utgGraphErrorLimit $=2.5$, utgMergeErrorRate $=0.030$, utgMergeErrorLimit $=3.25$, dovlErrorRate $=$ 0.1 , cnsErrorRate $=0.1$, cgwErrorRate $=0.1$, merSize $=22$, and doOverlapBasedTrimming $=1$. The initial Celera assembly had a total size of $602 \mathrm{Mb}$, N50 Contig size of $8,581 \mathrm{bp}$, and N50 scaffold size of $219 \mathrm{~kb}$ with 88,548 gaps $(18 \mathrm{Mb})$. The size distributions of the Celera contigs were plotted and the assembled contig revealed a contig coverage of approximately $33 \times$ (Additional file 1: Table S3 and Additional file 2: Figure S1).

\section{Error correction of PacbioRS reads}

The genome was sequenced using PacbioRS, which can generate continuous long reads (CLRs) of up to $10 \mathrm{~kb}$ in length, and can be used to upgrade draft genomes containing gaps using PBJelly (Ver. 12.9.14) [22]. However, 
CLRs show only $82.1 \%$ to $84.4 \%$ base accuracy [55]. Thus, error correction was performed using the command pacBioToCA [56] with the parameters -length 500, -partitions 200, -shortReads, -l NC, -t 20, and -s pacbio.spec. Illumina (50 $\times$ read coverage of genome) reads were used for correction. Illumina reads were trimmed using FASTX-Toolkit [56] with the parameters -t 20, -1 50, and -Q 33. Pacbio.spec files specified the parameters for overlapping Illumina and pacbio data for correction: utgErrorRate $=0.25$, utgErrorLimit tgErrorLcnsErrorRate $=0.25$, cgwErrorRate $=0.25$, ovlErrorRate $=$ 0.25 , and merSize $=10$. After correction, pacBio-corrected reads were analyzed using FastQC [57]. A total of 2,640,379 CLRs (7.6x read coverage of genome) were used for errorcorrection, which generated 2,415,333 error-corrected reads (2.3× read coverage of genome) (Additional file 1: Table S1). The average CLR length decreased from 1,819 to $969 \mathrm{bp}$. The resulting error-corrected CLRs were used for gap filling.

\section{Gap filling}

Gap filling was conducted in two stages. Initially, we closed gaps using the Gapfiller Ver.1.9 software with $116 \times$ trimmed Illumina reads with default settings $[23,58]$. The remaining gaps of the scaffold from Gapfiller were closed using error-corrected CLRs from PacbioRS using the PBJelly software (Ver. 12.9.14) with the parameter of minGap $=10$ [22]. Using Gapfiller, 18,400 gaps (2.3 Mb in length) were closed and 7,394 gaps $(3.0 \mathrm{Mb})$ were filled with error-corrected CLRs. A total of 25,794 gaps were closed (closed gap size of 5.3 Mbases). After gap filling, the number of scaffolds decreased from 11,622 to 8,155 and the N50 contig size increased from 8,518 bases to 11,563 bases (Additional file 1: Table S3).

\section{Repeat analysis}

We constructed a de novo repeat library using RepeatModeler (Ver. 1.0.3) [59], including the RECON (Ver. 1.07) [59] and RepeatScout (Ver. 1.0.5) [60] software, with default parameters. Consensus sequences and classification information for each repeat family were generated, and tandem repeats including simple repeats, satellites, and low complexity repeats were predicted using TRF [61].

\section{Assembly validation}

The $N$. coriiceps BAC library was obtained from the Children's Hospital Oakland Research Institute (BAC library ID, VMRC-19). We sequenced six BAC clones using GS-FLX and assemblies were performed using the Celera Assembler (Ver. 7.0). Six sequenced BAC clones were aligned to the assembled genome scaffolds using NUCmer (Ver. 3.07) with default settings. Mummerplot (Ver. 3.5) was used with the NUCmer delta file as input [62] (Additional file 2: Figure S2).

\section{Transcriptome assembly}

Total RNA from seven tissues (brain, skin, egg, kidney, muscle, stomach, and blood) was prepared using the Qiagen kit according to the manufacturer's instructions. The quality of total RNA was confirmed on an Agilent Bioanalyzer $^{\mathrm{Tm}}$. Library construction and sequencing were performed using DNAlink with an Illumina HiSeq 2000 System and PacbioRS. A total of 36,046 Mbases and 300 Mbases were obtained using the two methods, respectively (Additional file 1: Table S4). The transcriptome sequence reads were mapped to the $N$. coriiceps genome using the publicly available packages Bowtie (Ver. 0.12.9) [63,64], TopHat (Ver. 2.0.6) [65,66], and Cufflinks (Ver. 2.0.2) [67-69] (Additional file 1: Table S5). PacbioRS reads from each tissue (egg, skin, and muscle) were error-corrected with Illumina paired-end reads of mRNAs corresponding to each tissue [56] (see the Error correction of PacbioRS reads and Additional file 1: Table S5). Transcript assemblies with Cufflinks and error-corrected CLR were both used for gene annotation.

\section{Gene annotation (MAKER)}

We used MAKER2 for genome annotation [70]. MAKER is a portable and easily configurable genome annotation pipeline. Maker first identified repetitive elements using RepeatMasker (Ver. 3.3.0) [71]. This masked genome sequence was used for $a b$ initio gene prediction with the SNAP software [72], after which alignment of expressed sequence tags with BLASTn and protein information from tBLASTx were included. We used the de novo repeat library of $N$. coriiceps from RepeatModeler (Ver. 1.0.5) for RepeatMasker (Ver. 3.3.0); proteins from five fish species with data from Ensembl release 69 (D. rerio, G. aculeatus, T. rubripes, T. nigroviridis, and G. morhua) were included in the analysis. Transcriptome assembly results were used for expressed sequence tags. Next, MAKER polished the alignments using the program Exonerate, which provided integrated information to synthesize SNAP annotation. MAKER then selected and revised the final gene model considering all information. A total of 32,661 transcripts and 32,260 genes were predicted using MAKER in N. coriiceps, and 93,090 $a b$ initio gene predictions were generated. Additionally, 29,045 out of 32,260 genes were assigned preliminary functions based on automated annotation using Blast2Go (Ver. 2.6.0) [73].

\section{Non-coding RNA}

The Infernal software package (Ver. 1.1) [74] and CMs from the Rfam database [75] were used to identify noncoding RNAs in the $N$. coriiceps scaffolds (Additional file 1: Table S8). We identified putative tRNA genes using tRNAscan-SE (Ver. 1.21) [76]. tRNAscan-SE uses a covariance model $(\mathrm{CM})$ that scores candidates based 
on their sequence and predicted secondary structures (Additional file 1: Table S9).

\section{Ortholog analysis}

We identified orthologous groups using OrthoMCL (Ver. 2.0.5) [77], which generated a graphical representation of sequence relationships that was then divided into subgraphs using the Markov Clustering Algorithm (MCL) from multiple eukaryotic genomes [77]. We used the standard parameters and options of OrthoMCL for all steps. In this analysis, six fish genomes (D. rerio, G. aculeatus, $T$. rubripes, T. nigroviridis, G. morhua, and N. coriiceps) were used, with coding sequences collected from Ensemble release 69 except for $N$. coriiceps (Additional file 2: Figure S4). For $N$. coriiceps, the coding sequence from the MAKER annotation pipeline was used.

\section{Likelihood analysis of gene gain and loss}

To estimate the average gene gain/loss rate and to identify gene families that have undergone significant size changes, we used the program CAFE3.0 [26,27,29,78]. The phylogenetic tree of the species drawn with Timetree [79] was used for analysis. We performed the program using $P<0.05$, estimated birth $(\lambda)$ and death $(\mu)$ rates by using the program lambdamu with '-s' option. We calculated the number of gene gains and losses on each branch of the tree with the '- $t$ ' option. Using $P<0.0001$, we expect there to be approximately one significant result by chance and calculated the exact $P$ values for transitions over every branch. We called individual branches significant at $P<0.005$ [29].

\section{$\mathrm{dN} / \mathrm{dS}$ analysis}

We first identified orthologous groups using OrthoMCL for $\mathrm{dN} / \mathrm{dS}$ analysis. Six fish genomes (D. rerio, G. aculeatus, T. rubripes, T. nigroviridis, G. morhua, and N. coriiceps) were used for analysis, and coding sequences from five genomes were collected from Ensembl release 69. We identified 8,974 orthologous groups common to all six fish (Additional file 1: Table S10). To establish sets of othologs among six fish, the method of reciprocal best hits using BLASTp was used. Protein-coding sequences of orthologs were aligned using PRANK (Ver. 130820) under a codon model [80], and poor alignment sites were eliminated using Gblock (Ver. 0.91) under a codon model [81]. Poor alignment sequences were also eliminated (below 50\% similarity in length and $40 \%$ in identity). Codeml in the Phylogenetic Analysis by Maximum Likelihood (PAML) package (Ver. 4.7a) was used to estimate the $\mathrm{dN}$ (the rate of non-synonymous substitutions), dS (the rate of synonymous substitutions) and the ratio of $\mathrm{dN} / \mathrm{dS}$ using the branch model (model $=2$, NSsites $=$ 0 , fix_omega $=0)$ and basic model $($ model $=0$, NSsites $=$ 0 , fix_omega $=0$ ) under F3X4 codon frequency and codon sequence types [82]. The species tree was calculated by using PHYLIP's dnaml (Ver. 3.695). To identify whether the $d N / d S$ in each lineage is different from the rest of tree, a Likelihood Ratio Test (LRT) of branch model to basic model was performed, and false discovery rate (FDR) was used to control the $P$ values in multiple tests. Additionally, we performed a LRT of a branch model to a model of neutrality (model $=2$, NSsite $=0$, fix_omega $=1$ ) and FDR was also used to adjust the $P$ value [82]. Orthologs with $\mathrm{dS}>3$ or tanssition/tranversion ratio $>10$ were filtered. Finally, dN/dS of 5,039 single-copy gene orthologs for the six fish was determined.

\section{Functional analysis of rapidly evolving genes}

$\mathrm{dN}$ was considered as the indicator to distinguish whether a protein rapidly evolved or not, because highly expressed genes may result in underestimates of the synonymous substitution rate even with likelihood methods [32]. To investigate whether any functional categories were statistically over-represented among rapidly evolving $N$. coriiceps genes (comprising the fastest evolving $10 \%$ of total genes, 505 genes in all) in terms of $\mathrm{dN}$ [30,32], we applied AgriGO [31], a web-based tool for gene ontology analysis, with significant levels of $P=0.05$. Complete hierarchies of $\mathrm{GO}$ terms for each gene were examined.

\section{Gene expression under temperature stress}

$N$. coriiceps were transported in insulated containers with aerated sea water to the King Sejong Station, and were acclimated in large tanks circulating with fresh sea water at $+2.0 \pm 0.2^{\circ} \mathrm{C}$ at least 3 days prior to experiments. We prepared two other large tanks at $-2^{\circ} \mathrm{C}, 2^{\circ} \mathrm{C}$, and $4^{\circ} \mathrm{C}$ for cold stress, control, and heat stress, respectively. After acclimation, three groups of nine specimens each of $N$. coriiceps were kept in a cold tank, a normal tank, and a heated tank with aerated sea water. Three groups of three specimens of $N$. coriiceps each were sacrificed at 0,24 , and $48 \mathrm{~h}$ after stress. We then dissected each tissue (brain, skin, egg, kidney, muscle, and stomach) of $N$. coriiceps. Before dissection, blood samples were collected from the brachial vein using a sterile $3 \mathrm{~mL}$ syringe. Dissected tissues were lysed, immersed in RNAlater, and stored at $-70^{\circ} \mathrm{C}$ for future experiments.

For RNA-Seq experiments, we prepared mRNA from blood samples from three specimens of each individual sample at each temperature condition. Sequencing was performed with Illumina Hiseq 2000, and generated reads were trimmed using sickle (Ver. 1.2) with approximately 75 bases in length and approximately 20 in base quality (Additional file 1: Table S13). Trimmed reads of each tissue were mapped to the annotated scaffold of the N. coriiceps genome using TopHat (Ver. 2.0.6) [66], and differentially expressed genes were assessed using Cuffdiff (Ver. 2.0.2) [69]. Cuffdiff compares FPKM (fragments per 
kilobase of exon per million fragments mapped) values between each sample and calculates fold changes in expression for each gene based on statistical significance (cutoff, $P \leq 0.05$ ) (Additional file 1: Table S14 and Additional file 5).

\section{Tissue-specific gene expression}

Illumina paired-end reads of each tissue were mapped to the annotated scaffold of $N$. coriiceps genome using TopHat (Ver. 2.0.6) [66], and differentially expressed genes were assessed using Cuffdiff (Ver. 2.0.2) [69] (cutoff, $P \leq 0.05$ ).

\section{Statistical analysis}

Comparisons of multiple samples were made by an analysis of variances (ANOVA) with Bonferroni post hoc test. The Statistical Package for the Social Sciences software (SPSS) was used for analyses.

\section{Accession codes}

The $N$. coriicpes has been deposited at BioProject: 66471, and the whole-genome shotgun project has been deposited at DDBJ/EMBL/GenBank under accession AZAD00000000. This paper describes the first version, AZAD01000000. Raw RNA sequencing reads have been submitted to the NCBI Sequence Read Archive database (SRA091269).

\section{Additional files}

Additional file 1: Table S1. Statistics for each DNA library. Ten categories of DNA libraries with various insert sizes for three platform sequencers were constructed. Table S2: Insert size of each paired-end libraries. The range of paired-end insert sizes was estimated by mapping the reads onto the assembled genome sequence. Table S3: Statistics of genome assembly and gap filling. Table S4: Sequencing statistics of transcriptome analysis of each organ of N. coriiceps using two sequencer platforms. Table S5: Assembly results of transcriptome analysis of each organ of $\mathrm{N}$. coriiceps. Table S6: Genome annotation statics. Table S7: General statistics of gene in N. coriiceps. Table S8: Known repetitive and transposable elements in the $N$. coriiceps genome. Table S9: Number of tRNA in the N. coriiceps nuclear genome. Table S10: Shared orthologous gene clusters among six fishes. For genes with multiple alternative transcripts, the transcript with the best alignment was selected. Genes with lengths less than $100 \mathrm{bp}$ were discarded. Table S11: $\mathrm{GO}$ terms over-represented in dN/dS analysis. Table S12: Gene which is included in $\mathrm{GO}$ terms over-represented in $\mathrm{dN} / \mathrm{dS}$ analysis. Table S13: Sequencing reads used in analysis of RNA-Seq under stresses. Table S14: The result of RNA-seq. Detailed gene lists are shown in Tables S16 - S22. Table S15: Upregulated genes in blood under both cold and heat stress. Table S16: GO enrichment test in blood under heat stress. Table S17: GO enrichment test in blood under cold stress. Table S18: Top blood-specific genes and their transcript percentages in whole blood transcriptomes. Table S19: Downregulated genes in blood under cold stress. Table S20: Downregulated genes in blood under heat stress. Table S21: Downregulated genes in blood under both cold and heat stress. Table S22: Shared genes in downregulated group under heat and cold stress in the brain.

Additional file 2: Figure S1. Contig characteristics. (A) The contig length distribution shows that small size contig were incorporated into other contig. (B) Contig coverage. Figure S2: Comparison of the assembled genome with six BACs sequences. NUCmer alignments of the Celera genome assembly scaffolds ( $x$-axis) and BAC sequences (y-axis) ordered and oriented such that the largest hits cluster. Figure S3: Gene ontology (GO) distribution after BlastX analysis for N. coriiceps transcriptome sequences as grouped by biological process (red), cellular component (cyan), and molecular function (grey). Figure S4: Cladogram representing phylogenetic relationship between selected 5,039 orthologous genes of six fishes. Figure S5: Sequence alignment of heat shock factor-1. The sites required for post-translational modification were well-conserved except Ser303, corresponding to human HSF1 in PDSM in Antarctic fish (N. coriiceps, Dragon fish and Icefish). Figure S6: Significantly over-represented GO terms under heat and cold stress among upregulated genes from blood. Figure S7: Tissue-specific expression of genes related to the HSR. (A) HSR and UPR were confirmed to be downregulated with RNA-Seq analysis in whole blood sample. (B) FKBP, HSP70, and HSP40 genes were confirmed to be downregulated in blood samples more than other tissues in HSR. XBP1 gene, the representative transcription factor in UPR, was also downregulated in blood sample. Figure S8: Significantly over-represented GO terms under heat and cold stress among downregulated genes from blood and gene expression included in heme binding (GO:0020037). (A,B) Significantly over-represented GO terms among the downregulated genes from blood showed the similar GO terms hierarchy. Five GO terms were shared in both cold and heat stress. (C) Results of RNA-Seq studies show the expression-pattern of genes included in GO:00200037. Figure S9: Toxicity of nitric oxide and superoxide. Figure S10: Forty-one GO terms focused on seven GO terms in the gene ontology hierarchy.

Additional file 3: Significant contraction. Thirty-two families showing significantly difference in $N$. coriiceps lineage. The program CAFE3.0 was used to identify gene families that have undergone significant size changes using $P<0.0001$

Additional file 4: Rapid evolving gene. $d N$ was considered as the indicator to distinguish whether a protein rapidly evolved or not, and 505 genes comprising the fastest evolving $10 \%$ of total genes were selected with $\mathrm{dN}$. A total of 505 genes were denoted as $R$ in rapid evolving gene (column D), N. coriiceps lineage-specific genes were denoted as D, and genes under positive selection were denoted as PS.

Additional file 5: Gene expression under temperature stress.

\section{Competing interests}

The authors declare that they have no competing interests.

\section{Authors' contributions}

HP and HWD conceived the project; HP, SCS, JP, and HWD conceived and designed experiments and analyses; SJK, HL, MKK, JL, JHL, and SGL performed experiments; SCS, JEL, DE, and CWP conducted bioinformatics. All authors have read and approved the final version of this manuscript.

\section{Acknowledgements}

We would like to thank Harold H. Zakon for comments and discussion. This work was supported by an Antarctic Fish Genome Project grant (PE11150, HP) and Antarctic Organisms: Cold-adaptation Mechanism and Its Application grant (PE14070, HP) funded by the Korea Polar Research Institute (KOPRI). Additional Funding was provided by U.S. National Institutes of Health grant 5R01AG031922 (JHP and HWD) and by U.S. National Science Foundation grants OPP-0944517 and PLR-1247510 (HWD). This is contribution number 316 from the Northeastern University Marine Science Center.

\section{Author details}

'Division of Polar Life Sciences, Korea Polar Research Institute, Yeonsu-gu, Incheon 406-840, South Korea. ${ }^{2}$ Polar Sciences, University of Science \& Technology, Yuseong-gu, Daejeon 305-333, South Korea. ${ }^{3}$ Division of Biotechnology, Korea University, Sungbuk-gu, Seoul 406-840, South Korea. ${ }^{4}$ Fred Hutchinson Cancer Research Center, 1100 Fairview Avenue North, D4-100, Seattle, WA 98109-1024, USA. ${ }^{5}$ DNA Link, Inc, Songpa-gu, Seoul 138-736, South Korea. ${ }^{6}$ Department of Marine and Environmental Sciences, Marine Science Center, Northeastern University, Nahant, MA 01908, USA. ${ }^{7}$ Department of Biology, University of Oregon, Eugene, OR 97403, USA. ${ }^{8}$ Australian Centre for Plant Functional Genomic, School of Agriculture and Food Sciences, University of Queensland, St Lucia, QLD, Australia. ${ }^{9}$ School of Plant Biology, University of Western Australia, Crawley, WA 6009, Australia. 
Received: 7 March 2014 Accepted: 11 September 2014

Published online: 25 September 2014

\section{References}

1. Clarke A, Crame JA, Stromberg J-O, Barker P: The Southern Ocean benthic fauna and climate change: a historical perspective. Philos Trans $R$ Soc Lond B Biol Sci 1992, 338:299-309.

2. Eastman JT, Pratt D, Winn W: Antarctic Fish Biology: Evolution in a Unique Environment. San Diego, CA: Academic Press; 1993.

3. Chen L, DeVries AL, Cheng CH: Evolution of antifreeze glycoprotein gene from a trypsinogen gene in Antarctic notothenioid fish. Proc Natl Acad Sci US A 1997, 94:3811-3816.

4. Cheng $\mathrm{CH}$, Cziko PA, Evans CW: Nonhepatic origin of notothenioid antifreeze reveals pancreatic synthesis as common mechanism in polar fish freezing avoidance. Proc Natl Acad Sci U S A 2006, 103:10491-10496.

5. DeVries AL: The role of antifreeze glycopeptides and peptides in the freezing avoidance of Antarctic fishes. Comp Biochem Physiol B 1988, 90:611-621.

6. Detrich HW 3rd, Johnson KA, Marchese-Ragona SP: Polymerization of Antarctic fish tubulins at low temperatures: energetic aspects. Biochemistry 1989, 28:10085-10093.

7. Detrich HW 3rd, Parker SK, Williams RC Jr, Nogales E, Downing KH: Cold adaptation of microtubule assembly and dynamics. Structural interpretation of primary sequence changes present in the alpha- and beta-tubulins of Antarctic fishes. J Biol Chem 2000, 275:37038-37047.

8. Hofmann GE, Buckley BA, Airaksinen S, Keen JE, Somero GN: Heat-shock protein expression is absent in the Antarctic fish Trematomus bernacchii (family Nototheniidae). J Exp Biol 2000, 203:2331-2339.

9. Place SP, Hofmann GE: Comparison of Hsc70 orthologs from polar and temperate notothenioid fishes: differences in prevention of aggregation and refolding of denatured proteins. Am J Physiol Regul Integr Comp Physiol 2005, 288:R1195-R1202.

10. Place SP, Zippay ML, Hofmann GE: Constitutive roles for inducible genes: evidence for the alteration in expression of the inducible hsp70 gene in Antarctic notothenioid fishes. Am J Physiol Regul Integr Comp Physiol 2004, 287:R429-R436.

11. Romisch K, Collie N, Soto N, Logue J, Lindsay M, Scheper W, Cheng CH: Protein translocation across the endoplasmic reticulum membrane in cold-adapted organisms. J Cell Sci 2003, 116:2875-2883.

12. Ruud JT: Vertebrates without erythrocytes and blood pigment. Nature 1954, 173:848-850.

13. Sidell BD, O'Brien KM: When bad things happen to good fish: the loss of hemoglobin and myoglobin expression in Antarctic icefishes. J Exp Biol 2006, 209:1791-1802.

14. Eastman JT: The nature of the diversity of Antarctic fishes. Polar Biol 2005, 28:93-107.

15. Eastman JT, Hubold G: The fish fauna of the Ross Sea, Antarctica. Antarctic Sci 1999, 11:293-304.

16. Andriashev A: A general review of the Antarctic bottom fish fauna. In Proceedings of the Fifth Congress of European Ichthyologists, Stockholm: 1985 Edited by Kullander SO, Fernholm B. Stockholm: Swedish Museum of National History; 1985:357-372.

17. Egginton S: Blood rheology of Antarctic fishes: viscosity adaptations at very low temperatures. J Fish Biol 1996, 48:513-521.

18. Hernandez-Blazquez FJ, Guerra RR, Kfoury JR Jr, Bombonato PP, Cogliati B, da Silva JRMC: Fat absorptive processes in the intestine of the Antarctic fish Notothenia coriiceps (Richardson, 1844). Polar Biol 2006, 29:831-836.

19. Johnston IA, Fernández DA, Calvo J, Vieira VL, North AW, Abercromby M, Garland T: Reduction in muscle fibre number during the adaptive radiation of notothenioid fishes: a phylogenetic perspective. J Exp Biol 2003, 206:2595-2609.

20. Gon O, Heemstra PC: (Eds): Fishes of the Southern Ocean, Volume 1 Grahamstown: JLB Smith Institute of Ichthyology; 1990.

21. Myers EW, Sutton GG, Delcher AL, Dew IM, Fasulo DP, Flanigan MJ, Kravitz SA, Mobarry CM, Reinert KH, Remington KA, Anson EL, Bolanos RA, Chou $\mathrm{HH}$, Jordan CM, Halpern AL, Lonardi S, Beasley EM, Brandon RC, Chen L, Dunn PJ, Lai Z, Liang Y, Nusskern DR, Zhan M, Zhang Q, Zheng X, Rubin GM, Adams MD, Venter JC: A whole-genome assembly of Drosophila. Science 2000, 287:2196-2204.

22. English AC, Richards S, Han Y, Wang M, Vee V, Qu J, Qin X, Muzny DM, Reid JG, Worley KC: Mind the gap: upgrading genomes with Pacific Biosciences RS long-read sequencing technology. PLoS One 2012, 7:e47768.
23. Nadalin F, Vezzi F, Policriti A: GapFiller: a de novo assembly approach to fill the gap within paired reads. BMC Bioinform 2012, 13:S8.

24. Cantarel BL, Korf I, Robb SM, Parra G, Ross E, Moore B, Holt C, Alvarado AS, Yandell M: MAKER: an easy-to-use annotation pipeline designed for emerging model organism genomes. Genome Res 2008, 18:188-196.

25. Lynch M, Conery JS: The evolutionary fate and consequences of duplicate genes. Science 2000, 290:1151-1155.

26. Demuth JP, De Bie T, Stajich JE, Cristianini N, Hahn MW: The evolution of mammalian gene families. PLoS One 2006, 1:e85.

27. Hahn MW, De Bie T, Stajich JE, Nguyen C, Cristianini N: Estimating the tempo and mode of gene family evolution from comparative genomic data. Genome Res 2005, 15:1153-1160.

28. De Bie T, Cristianini N, Demuth JP, Hahn MW: CAFE: a computational tool for the study of gene family evolution. Bioinformatics 2006, 22:1269-1271.

29. Hahn MW, Han MV, Han S-G: Gene family evolution across 12 Drosophila genomes. PLoS Genet 2007, 3:e197.

30. Castillo-Davis Cl, Kondrashov FA, Hartl DL, Kulathinal RJ: The functional genomic distribution of protein divergence in two animal phyla: coevolution, genomic conflict, and constraint. Genome Res 2004, 14:802-811.

31. Du Z, Zhou X, Ling Y, Zhang Z, Su Z: agriGO: a GO analysis toolkit for the agricultural community. Nucleic Acids Res 2010, 38:W64-W70.

32. Dunn KA, Bielawski JP, Yang Z: Substitution rates in Drosophila nuclear genes: implications for translational selection. Genetics 2001, 157:295-305.

33. Mark FC, Lucassen M, Strobel A, Barrera-Oro E, Koschnick N, Zane L, Patarnello T, Pörtner HO, Papetti C: Mitochondrial function in Antarctic nototheniids with ND6 translocation. PLoS One 2012, 7:e31860.

34. Strobel A, Graeve M, Poertner HO, Mark FC: Mitochondrial acclimation capacities to ocean warming and acidification are limited in the Antarctic Nototheniid fish, Notothenia rossii and Lepidonotothen squamifrons. PLoS One 2013, 8:e68865.

35. Weinstein R, Somero G: Effects of temperature on mitochondrial function in the Antarctic fish Trematomus bernacchii. J Comp Physiol B 1998, 168:190-196.

36. Buckley BA, Place SP, Hofmann GE: Regulation of heat shock genes in isolated hepatocytes from an Antarctic fish, Trematomus bernacchii. J Exp Biol 2004, 207:3649-3656.

37. Chen Z, Cheng CH, Zhang J, Cao L, Chen L, Zhou L, Jin Y, Ye H, Deng C, Dai Z, Xu Q, Hu P, Sun S, Shen Y, Chen L: Transcriptomic and genomic evolution under constant cold in Antarctic notothenioid fish. Proc Natl Acad Sci U S A 2008, 105:12944-12949.

38. Franks F: Protein destabilization at low temperatures. Adv Protein Chem 1995, 46:105-139.

39. Todgham AE, Hoaglund EA, Hofmann GE: Is cold the new hot? Elevated ubiquitin-conjugated protein levels in tissues of Antarctic fish as evidence for cold-denaturation of proteins in vivo. J Comp Physiol B 2007, 177:857-866.

40. Bettencourt BR, Hogan CC, Nimali M, Drohan BW: Inducible and constitutive heat shock gene expression responds to modification of Hsp70 copy number in Drosophila melanogaster but does not compensate for loss of thermotolerance in Hsp70 null flies. BMC Biol 2008, 6:5.

41. Akerfelt M, Morimoto RI, Sistonen L: Heat shock factors: integrators of cell stress, development and lifespan. Nat Rev Mol Cell Biol 2010, 11:545-555.

42. Hietakangas V, Ahlskog JK, Jakobsson AM, Hellesuo M, Sahlberg NM, Holmberg Cl, Mikhailov A, Palvimo JJ, Pirkkala L, Sistonen L: Phosphorylation of serine 303 is a prerequisite for the stress-inducible SUMO modification of heat shock factor 1. Mol Cell Biol 2003, 23:2953-2968

43. Hietakangas V, Anckar J, Blomster HA, Fujimoto M, Palvimo JJ, Nakai A, Sistonen L: PDSM, a motif for phosphorylation-dependent SUMO modification. Proc Natl Acad Sci U S A 2006, 103:45-50.

44. Kline MP, Morimoto RI: Repression of the heat shock factor 1 transcriptional activation domain is modulated by constitutive phosphorylation. Mol Cell Biol 1997, 17:2107-2115.

45. Somero GN, DeVries AL: Temperature tolerance of some Antarctic fishes. Science 1967, 156:257-258.

46. Gulevsky AK, Relina LI: Molecular and genetic aspects of protein cold denaturation. Cryo Letters 2013, 34:62-82.

47. Bernales S, Papa FR, Walter P: Intracellular signaling by the unfolded protein response. Annu Rev Cell Dev Biol 2006, 22:487-508. 
48. Calfon M, Zeng H, Urano F, Till JH, Hubbard SR, Harding HP, Clark SG, Ron D: IRE1 couples endoplasmic reticulum load to secretory capacity by processing the XBP-1 mRNA. Nature 2002, 415:92-96.

49. Kaufman RJ: Stress signaling from the lumen of the endoplasmic reticulum: coordination of gene transcriptional and translational controls. Genes Dev 1999, 13:1211-1233.

50. Bargelloni L, Marcato S, Patarnello T: Antarctic fish hemoglobins: evidence for adaptive evolution at subzero temperature. Proc Natl Acad Sci 1998 , 95:8670-8675

51. D'avino R, di Prisco G: Hemoglobin from the Antarctic fish Notothenia coriiceps neglecta. Eur J Biochem 1989, 179:699-705.

52. Westerheide SD, Anckar J, Stevens SM Jr, Sistonen L, Morimoto RI: Stress-inducible regulation of heat shock factor 1 by the deacetylase SIRT1. Science 2009, 323:1063-1066.

53. Zukowski S-SC: Blood of antarctic fishes : Notothenia rossi marmorata Fischer and Notothenia neglecta Nybelin. Pol Polar Res 1980, 1:103-108.

54. Gordon A, Hannon GJ: Fastx-toolkit: FASTQ/A short-reads preprocessing tools. [http://hannonlab.cshl.edu/fastx_toolkit]

55. Rasko DA, Webster DR, Sahl JW, Bashir A, Boisen N, Scheutz F, Paxinos EE, Sebra R, Chin CS, lliopoulos D, Klammer A, Peluso P, Lee L, Kislyuk AO, Bullard J, Kasarskis A, Wang S, Eid J, Rank D, Redman JC, Steyert SR, Frimodt-Møller J, Struve C, Petersen AM, Krogfelt KA, Nataro JP, Schadt EE, Waldor MK: Origins of the E. coli strain causing an outbreak of hemolytic-uremic syndrome in Germany. N Engl J Med 2011, 365:709-717.

56. Koren S, Schatz MC, Walenz BP, Martin J, Howard JT, Ganapathy G, Wang Z Rasko DA, McCombie WR, Jarvis ED, Phillippy AM: Hybrid error correction and de novo assembly of single-molecule sequencing reads. Nat Biotechnol 2012, 30:693-700.

57. FastQC. [http://www.bioinformatics.babraham.ac.uk/projects/fastqc]

58. Boetzer M, Pirovano W: Toward almost closed genomes with GapFiller. Genome Biol 2012, 13:R56.

59. Bao Z, Eddy SR: Automated de novo identification of repeat sequence families in sequenced genomes. Genome Res 2002, 12:1269-1276.

60. Price $A L$, Jones NC, Pevzner PA: De novo identification of repeat families in large genomes. Bioinformatics 2005, 21:i351-i358.

61. Benson G: Tandem repeats finder: a program to analyze DNA sequences. Nucleic Acids Res 1999, 27:573-580.

62. Delcher AL, Salzberg SL, Phillippy AM: Using MUMmer to identify similar regions in large sequence sets. Curr Protoc Bioinformatics 2003, Chapter 10:Unit 10.3 .

63. Bowtie: An ultrafast memory-efficient short read aligner. [http://bowtie-bio. sourceforge.net/index.shtml]

64. Langmead B, Trapnell C, Pop M, Salzberg SL: Ultrafast and memory-efficient alignment of short DNA sequences to the human genome. Genome Biol 2009, 10:R25.

65. TopHat: A spliced read mapper for RNA-Seq. [http://ccb.jhu.edu/software/ tophat/index.shtml]

66. Trapnell C, Pachter L, Salzberg SL: TopHat: discovering splice junctions with RNA-Seq. Bioinformatics 2009, 25:1105-1111.

67. Cufflinks: Transcript assembly, differential expression, and differential regulation for RNA-Seq. [http://cufflinks.cbcb.umd.edu]

68. Roberts A, Pimentel H, Trapnell C, Pachter L: Identification of novel transcripts in annotated genomes using RNA-Seq. Bioinformatics 2011, 27:2325-2329

69. Trapnell C, Hendrickson DG, Sauvageau M, Goff L, Rinn JL, Pachter L. Differential analysis of gene regulation at transcript resolution with RNA-seq. Nat Biotechnol 2013, 31:46-53.

70. Holt C, Yandell M: MAKER2: an annotation pipeline and genome-database management tool for second-generation genome projects. BMC Bioinform 2011, 12:491.

71. Smit AFA HR, Green P: RepeatMasker Open-3.0. 1996-2004. [http://www. repeatmasker.org/]

72. Korf I: Gene finding in novel genomes. BMC Bioinform 2004, 5:59.

73. Conesa A, Gotz S, Garcia-Gomez JM, Terol J, Talon M, Robles M: Blast2GO: a universal tool for annotation, visualization and analysis in functional genomics research. Bioinformatics 2005, 21:3674-3676.

74. Nawrocki EP, Kolbe DL, Eddy SR: Infernal 1.0: inference of RNA alignments. Bioinformatics 2009, 25:1335-1337.

75. Gardner PP, Daub J, Tate J, Moore BL, Osuch IH, Griffiths-Jones S, Finn RD, Nawrocki EP, Kolbe DL, Eddy SR, Bateman A: Rfam: Wikipedia, clans and the "decimal" release. Nucleic Acids Res 2011, 39:D141-D145.
76. Lowe TM, Eddy SR: tRNAscan-SE: a program for improved detection of transfer RNA genes in genomic sequence. Nucleic Acids Res 1997, 25:955-964.

77. Li L, Stoeckert CJ Jr, Roos DS: OrthoMCL: identification of ortholog groups for eukaryotic genomes. Genome Res 2003, 13:2178-2189.

78. CAFE: Computational analysis of (gene) family evolution. [www.bio. indiana.edu/ hahnlab/Software.html]

79. Hedges SB, Dudley J, Kumar S: TimeTree: a public knowledge-base of divergence times among organisms. Bioinformatics 2006, 22:2971-2972.

80. Loytynoja A, Goldman N: An algorithm for progressive multiple alignment of sequences with insertions. Proc Natl Acad Sci U S A 2005, 102:10557-10562.

81. Castresana J: Selection of conserved blocks from multiple alignments for their use in phylogenetic analysis. Mol Biol Evol 2000, 17:540-552.

82. Yang Z: PAML 4: phylogenetic analysis by maximum likelihood. $\mathrm{Mol}$ Biol Evol 2007, 24:1586-1591.

doi:10.1186/s13059-014-0468-1

Cite this article as: Shin et al:: The genome sequence of the Antarctic bullhead notothen reveals evolutionary adaptations to a cold environment. Genome Biology 2014 15:468.

\section{Submit your next manuscript to BioMed Central and take full advantage of:}

- Convenient online submission

- Thorough peer review

- No space constraints or color figure charges

- Immediate publication on acceptance

- Inclusion in PubMed, CAS, Scopus and Google Scholar

- Research which is freely available for redistribution

Submit your manuscript at www.biomedcentral.com/submit
C) Biomed Central 